\title{
Session 1.3: Health Protection and Disease Prevention: A Critical Review of Experience
}

\author{
Ms. Joanna Merlin-Scholtes (Chair); ${ }^{1}$ Dr. Jai Narain (Rapporteur); ${ }^{2}$ \\ Dr. Supamit Chunsuttiwat (Speaker 1); ${ }^{3}$ Dr. Caroline Hyde-Price (Speaker 2); ${ }^{4}$ \\ Dr. Philippe Francois Dubois (Speaker 3); ${ }^{5}$ Dr. Eigil Sorensen (Discussant) ${ }^{6}$
}

1. United Nations Resident Coordinator, Thailand

2. Coordinator, HIV/AIDS, Tuberculosis and Other Communicable Diseases,

World Health Organization (WHO)/South East Asia Region

3. Senior Expert in Preventive Medicine, Department of Disease Control, Ministry of Public Health, Thailand

4. Head of International Office, Health Protection Agency, United Kingdom

5. Communicable Disease Surveillance and Response, WHO Headquarters, Geneva, Switzerland

6. WHO Representative, WHO/

Democratic People's Republic of Korea (DPRK)

\begin{abstract}
Keywords: communicable disease; coordination; effectiveness; health; international; laboratory support; lessons learned; needs assessment; nutrition; outbreak; preparedness; prevention; protection; sanitation; Thailand; Tsunami; water-borne; World Health Organization (WHO)
\end{abstract}
Abbreviations:
GOARN = Global Outbreak Alert and
Response Network
HPA $=$ Health Protection Agency
$\mathrm{MPH}=$ Ministry of Public Health
$\mathrm{NGO}=$ non-governmental organization
UK $=$ United Kingdom
$\mathrm{WHO}=$ World Health Organization

Web publication: 17 November 2005

\begin{abstract}
This is a summary of the presentations and discussion of Health Protection and Disease Prevention of the Conference, Health Aspects of the Tsunami Disaster in Asia, convened by the World Health Organization (WHO) in Phuket, Thailand, 04-06 May 2005. The topics discussed included issues related health protection and disease prevention as pertaining to the responses to the damage created by the Tsunami. It is presented in the following major sections: (1) key questions; (2) national perspectives; (3) an international perspective; (4) laboratory aspects in disease surveillance; and (5) partnership.
\end{abstract}

Merlin-Scholtes J, Narain J, Chunsuttiwat S, Hyde-Price C, Dubois PF, Sorensen E: Health protection and disease prevention: A critical review of experience. Prehosp Disast Med 2005;20(6):385-388.

\section{Background}

The Tsunami had devastating effects on the health and well-being of the affected communities. During the immediate phase of the crisis, relief efforts provided shelter and basic services to many of the survivors. The retrieval and safe disposal of dead bodies also was critical during this phase. The primary needs following the immediate phase included: (1) improving housing and shelter; (2) enhancing nutrition; (3) providing access to safe drinking water; (4) maintaining sanitation services; and (4) strengthening healthcare systems. The provision of sanitary services was essential because in such situations, water-borne, diarrheal diseases, like cholera and bacillary dysentery, are immediate risks to the affected population. The need for immunizations, reproductive and mental health, and environmental sanitation services also were among the identified priorities. During the intermediate and long-term phases, there also was the need for re-establishing health systems, which included rebuilding public health, laboratory, and communication facilities.

Experiences, thus far, show that coordinated and sustained efforts are critical for the provision of effective emergency responses and for the delivery of basic health services. At the time of the Conference, the World Health Organization (WHO) continued to serve in a leadership capacity in the health-sector responses, along with national governments and the local and international communities to re-establish vital social services, including health systems and services. The guiding principles included: (1) coordinating various partners in the implementation of actions; (2) building on existing structures and resources; (3) increasing synergy with national systems to improve epidemic preparedness, health promotion, and the strengthening of health systems; (4) enhancing public-private partnerships; (5) documenting experiences and lessons; (6) increasing accountability and transparency for implementation and reporting on the status of activities; and (7) ensuring local ownership and participation for all activities.

Key Questions

This panel addressed the: (1) appropriateness; (2) adequacy; (3) effectiveness; (4) efficiency; and (5) "connectedness" of both the technical and organiza- 


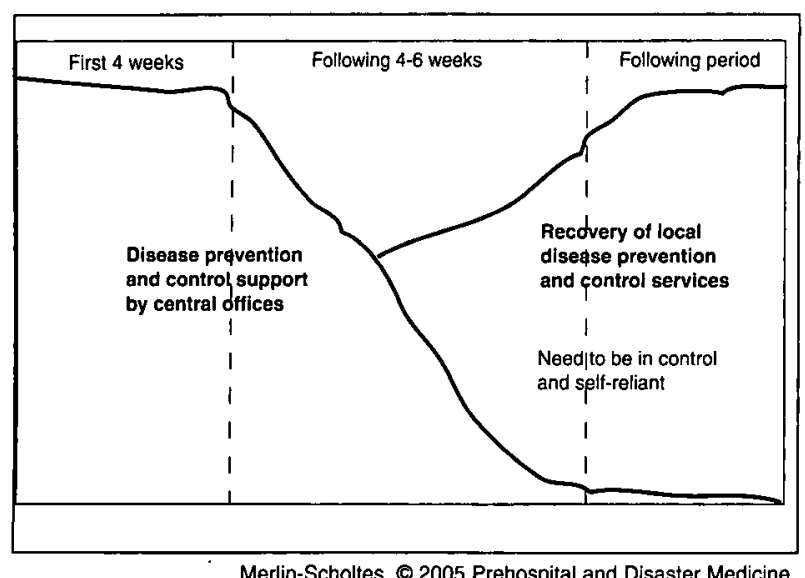

Figure 1-Planned phasing of disease prevention and control support to affected provinces in Thailand

tional aspects of national and international interventions for health protection and disease prevention of the affected members of society. The following key questions were discussed:

1. Needs assessment: How sound were the initial and ongoing health assessments of the disaster?;

2. Coordination: How are stakeholders cooperating and coordinating with each other, (which includes how resources are being mobilized and utilized)?;

3. Gap filling: How are population needs being met, and with what results?; and

4. Capacity building: How are country capacities being strengthened so as to be better prepared for future disasters?

In the context of improving the international health response to disasters, some of the specific issues and challenges for health protection and disease prevention that were addressed included:

1. Establishing and sustaining early warning systems;

2. Networking referrals and laboratory capacity;

3. Rebuilding health infrastructure;

4. The role of the private for-profit, non-governmental organizations (NGOs), and other healthcare providers;

5. Coordinating disease surveillance with partners;

6. Addressing mental health needs;

7. Addressing food and nutrition;

8. Working with a variety of partners (in the water and sanitation fields); and

9. Emphasizing the critical importance of health promotion and risk communication.

\section{Discussion}

\section{National perspective}

Center for Disease Control-The experiences obtained from the health protection and disease prevention activities in Thailand were outlined during the presentations and discussions. On 27 December 2004, one day after the Tsunami struck six provinces in Thailand, an Operations Center for Disease Control was established by the Ministry of Public Health (MPH). The organization of the Center was based on the existing severe acute respiratory syndrome (SARS) and avian influenza Operation Center. This

\begin{tabular}{|l|c|c|c|}
\hline $\begin{array}{c}\text { Category of tests and } \\
\text { specimens }\end{array}$ & $\begin{array}{c}\text { Number of } \\
\text { specimens tested }\end{array}$ & $\mathbf{n}$ & $\begin{array}{c}\text { Positive } \\
\text { finding (\%) }\end{array}$ \\
\hline Bacterial contamination \\
\hline Processed food & 1,179 & 47 & 4 \\
\hline Fresh seafood & 101 & 5 & 5 \\
\hline Bottled water & 453 & 73 & 16 \\
\hline Ice & 74 & 43 & 58 \\
\hline Heavy metal contamination & 12 & 0 & 0 \\
\hline Fresh seafood & 6 & 0 & 0 \\
\hline Sea water & \multicolumn{3}{|c|}{} \\
\hline \multicolumn{4}{|c|}{ Merlin-Scholtes @ 2005 Prehospital and Disaster Medicine }
\end{tabular}

Table 1-Food, water, and environment surveillance in six tsunami-affected provinces, 01-27 January 2005 in Thailand $(\mathrm{n}=$ number of specimens collected)

Center served as the base for all planning and coordination for all of the activities that related to communicable disease surveillance, outbreak investigation, and public information. Following the Tsunami, an initial and ongoing assessment of the critical needs was conducted, and the possible occurrences of various communicable diseases that likely would occur were anticipated. This helped in the planning of disease prevention support from the MPH to the affected provinces (Figure 1).

Contamination-Teams were established and assigned responsibilities for the surveillance and responses for vector control and sanitation support-each team was assigned specific roles in these processes. Surveillance data were collected from local hospitals, health centers, and other medical facilities. These data were processed and interpreted, and daily summary reports were sent to the provincial health centers. To ensure food and potable water safety, samples were collected and tested for bacterial contamination. The results of these analyses are in Table 1 . A total of 1,825 specimens were tested. Specimens from ice yielded the greatest proportion of positive results $(58 \%$ were positive). Sixteen percent of the bottled water also tested positive. Contamination of heavy metals was not present in any of the samples. Besides a few sporadic cases, no major outbreaks of communicable diseases were reported within the first four months following the Tsunami.

Lessons Learned-During this period in Thailand, many lessons were learned. The leadership provided by the Government and MPH was the key factor for the mobilization of multi-sectoral coordination and needed resources. The field coordination and control system employed functioned well using the WHO guidelines. This system contributed to disease control and rapid recovery (Figure 2).

However, experience also showed that Thailand was not prepared for such a major crisis as the Tsunami, as it lacked a preparedness plan, stockpiles of essential items, and had a limited capacity for international coordination and communication. As a result of these experiences, there is an identified need in Thailand to further: 


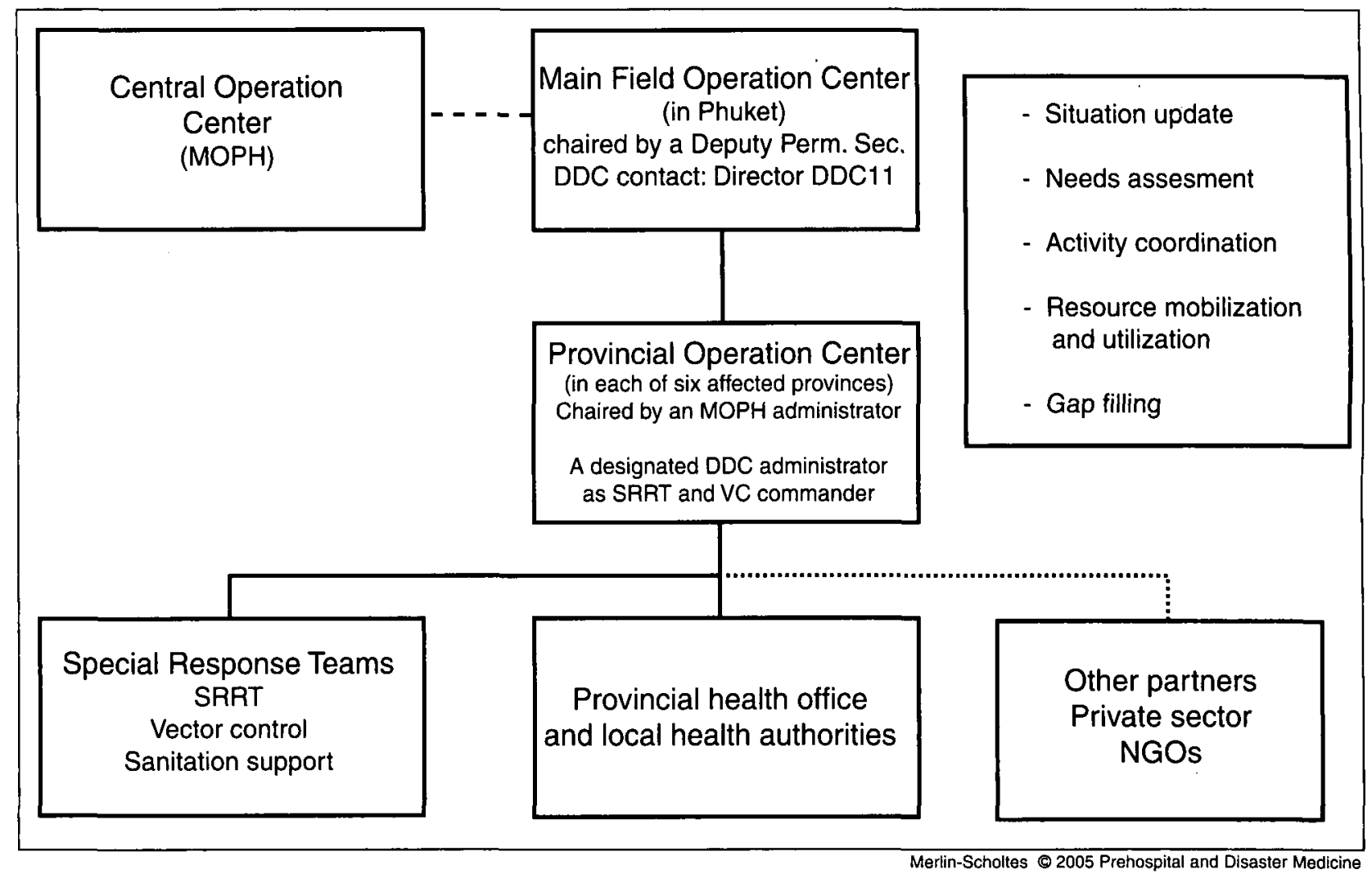

Figure 2-Field coordination in Thailand (DDC = Bureau of Communicable Disease; $\mathrm{MOPH}=$ Ministry of Public Health; NGO = non-governmental organization; SRRT $=$ Special Response Relief Team; VC $=$ Vesctor Control)

1. Improve the organizational aspects of the disaster health management by re-establishing a standing office for coordination of preparedness and response in the $\mathrm{MPH}$;

2. Enhance preparedness planning;

3. Enhance capacity building, including training;

4. Stockpile essential items; and

5. Improve risk communication using public education and communication channels.

At the international level, the MPH could benefit by increasing its collaboration in the development and implementation of an early warning system, in building international stockpiles of essential items, and by improving its communication with the international community.

\section{International perspectives}

An international perspective, based on a critical review of experiences, was presented on behalf of the Health Protection Agency (HPA). The HPA is a specialized, independent, public organization established by the government of the United Kingdom (UK) in 2003. It coordinates and provides health protection services across the UK and deals with all health protection threats. Health protection initiatives focus on disease prevention and reducing the adverse effects of chemical and biological hazards. There are eight themes that are essential to health protection:

1. Intellegence and needs assessments-Good, objective intelligence and needs assessments are key to obtaining a clear picture of the needs. The needs detected were overwhelming in the context of the Tsunami.
Based on the WHO's needs assessments, the HPA assigned its staff to assist in health promotion and disease prevention efforts, including those in Banda Aceh, and provided advice on the management of dead bodies.

2. Infectious diseases-Infectious diseases pose a major threat. Although there were no major disease outbreaks reported from any of the Tsunami-affected countries in Asia following the Tsunami, outbreaks were expected, given the conditions in which the affected people had to live. There was some debate as to whether public health professionals exaggerated the potential risk of outbreaks at the beginning of the responses to the disaster. The plenary session presenters argued that, in the past, many disasters, such as floods and drought, have witnessed outbreaks of infectious diseases including diarrhea, malaria, and measles. Therefore, it was reasonable to expect communicable disease outbreaks also would occur post-Tsunami. Moreover, people were displaced, living in over-crowded conditions with poor sanitation and water, thereby creating an environment conducive to the easy and rapid spread of communicable diseases.

Many health events, such as cases of measles and dengue, have been investigated as part of an early warning system in the Tsunami-affected areas. Verified and preventive measures were taken, which contributed to the prevention and/or limitation of 
further infectious disease transmission. It is clear that dead bodies do not lead to any infectious disease outbreaks and efforts to dispel this myth were made during the response.

3. Surveillance and early response-Good surveillance and early response saves lives. Surveillance and response in disease control requires a multi-sectoral response and a high level of political commitment. Clearly, health is not the responsibility of the health ministry alone, and health cannot be left only to the health ministry.

4. Interventions and experience-Evidence-based interventions and the use of previous experiences are vital. Experience shows that epidemics do not occur spontaneously during a disaster and dead bodies do not lead to outbreaks of diseases. The key to preventing diseases is to improve sanitary conditions and public education.

5. Psychological effects-Psychological sequelae are inevitable among victims and responders, but can be reduced.

6. Existing structures-Build on what exists already rather than create new structures in order to ensure the sustainability of the response.

7. Experience and practice-Learn from experience and practice beforehand. This can help when similar events occur frequently.

8. Anticipate and prepare-Anticipate the expected, but be prepared for surprises. For example, many of the organizations that responded to the Tsunami disaster did not anticipate or prepare for responding to this health situation and had no stockpiles of tetanus antitoxin in their supplies.

These eight themes could form some of the guiding principles in planning and responding to disasters, and could help in protecting the health of the affected populations.

\section{Laboratory Aspects}

Laboratory support often is not given necessary importance while planning for surveillance and responses. Clinical laboratory studies can assist with the diagnosis of health conditions and guide treatment options through monitoring of resistance patterns. However, in many countries, clinical laboratories are not well developed, and there is a need for a systematic strengthening of the clinical laboratory infrastructure. Such a process is essential for disease surveillance and control, and for responding appropriately to the disaster situation when people suffer from communicable diseases.

The quality of clinical laboratory services is important in disaster work. Capacity building of clinical laboratory workers in both the public and private sectors is critical. In addition, they must participate in proficiency testing. Strengthening health infrastructure, along with monitoring and supervision, are critical aspects of disaster management.

One practical problem faced in the field is how to store laboratory specimens and transport them properly to the appropriate laboratories. Simple field manuals that can be disseminated to personnel in the field to assist them in this important area currently are available.

The capabilities and capacities of the diagnostic laboratories should be done. Except for in biosafety and reporting, the capabilities of the clinical laboratories are greater than those in the periphery. Of substantial concern are the low marks for biosafety and hygiene $(<25 \%)$. Ultimately, greater recognition of the importance of the role of clinical laboratory services in communicable disease surveillance and responses, and building infrastructure and capacity building will contribute to improved communicable disease control during disasters.

\section{Partnerships}

Another issue discussed related to the role of partnerships during the disaster including involvement of the Global Outbreak Alert and Response Network (GOARN) coordinated by the WHO. A major, positive outcome of the Tsunami was the outpouring of support from the local, national, and international community. The GOARN partners, WHO staff and consultants, the (US) Centers for Disease Control and Prevention, and experts from many countries working hand-in-hand with national health personnel all contributed to establishing emergency surveillance, the early warning system, and the outbreak alert system, and the rapid responses to any communicable disease events. The role played by the public health professionals from the local level to the international level ultimately was responsible in ensuring that no outbreaks of communicable diseases occurred.

The role of the media in highlighting the disaster, its magnitude, its intensity, and the visual images of the event and its impact was instrumental in mobilizing the enormous international response to this large-scale event.

\section{Conclusion}

Surveillance and monitoring for diseases are essential activities in times of disaster. The organization infrastructure, capabilities, and capacities of the clinical laboratories must be strengthened. 